\title{
AVALIAÇÃo DE MÉTOdOS DE MEDIÇÃO DE ALTURA EM FLORESTAS NATURAIS ${ }^{1}$
}

\author{
Gilson Fernandes da Silva ${ }^{2}$, Rafaella De Angeli Curto ${ }^{3}$, Carlos Pedro Boechat Soares ${ }^{4}$ e Ludmila de
} Castro Piassi ${ }^{5}$

\begin{abstract}
RESUMO - Este trabalho teve como objetivo comparar métodos de medição de altura total de árvores em floresta natural. Foram comparadas alturas totais medidas pelo Hipsômetro Vertex com as obtidas por escalada das árvores. Também foi testado o método de estimação visual, com e sem treinamento, como alternativa mais prática e de menor custo. Os métodos testados foram avaliados em três classes de altura: 1 - (15-25 m); 2 - (25-35 m); e 3 - (> 35 m). Ao final, concluiu-se que o aumento na altura da árvore comprometeu a precisão da estimação pelos métodos avaliados, sendo mais evidente na estimação visual sem treinamento. A única diferença estatística a 5\% de probabilidade, pelo teste t, entre as médias das alturas para os métodos avaliados e as médias das alturas obtidas por escalada, nas diferentes classes, ocorreu na estimação visual com treinamento na classe 2. As estatísticas Desvio Médio ( $D M)$, Média das Diferenças Absolutas $(M D)$ e Desvio-Padrão das Diferenças $(D P D)$ indicaram maior precisão na estimação da altura para a estimação visual com treinamento, seguida pelo Vertex e pela estimação visual sem treinamento.
\end{abstract}

Palavras-chave: Dendrometria, Hipsometria e Métodos de medição.

\section{EVALUATION OF HEIGHT MEASUREMENT METHODS IN NATURAL FORESTS}

\begin{abstract}
The objective of this work was to compare methods of tree height measure in natural foress. Total heights measured by hypsometer Vertex were compared with those obtained by escalade of the trees. The method of visual estimate was also tested, with and without training, as a more practical alternative and of slower cost. The tested methods were evaluated for three height classes: 1 - (15-25 meters); 2 - (25-35 meters) and; 3 - (> 35 meters). At the end, it was concluded that the increase in the height of the trees reduced precision of the estimate for the appraised methods, being more evident in the visual estimate without training. The only statistical difference, at the level of $5 \%$ of probability for the test $t$, among the averages of heights for the appraised methods and the averages of the heights obtained by escalade, in the different classes, occurred in the visual estimate with training in the class 2. The statistics Average Deviation (DM), Average of the Absolute Differences (MD) and Standard Deviation of the Differences (DPD) indicated larger precision in the estimate of the height for the visual estimate with training, following Vertex and visual estimate without training.
\end{abstract}

Keywords: Forest mensuration, Hypsometer and Measurement methods.

\footnotetext{
${ }^{1}$ Recebido em 17.09.2008 e aceito para publicação em 15.03.12

${ }^{2}$ Departamento de Engenharia Florestal, Universidade Federal do Espírito Santo, UFES, Brasil. E-mail: <gilson.silva@pq.cnpq.br>.

${ }^{3}$ Engenheira Florestal, Doutoranda em Engenharia Florestal, Universidade Federal do Paraná, UFPR, Brasil. E-mail: <rafaellacurto@yahoo.com.br>.

${ }^{4}$ Departamento de Engenharia Florestal, Universidade Federal de Viçosa, UFV, Brasil. E-mail: <csoares@ufv.br>.

${ }^{5}$ Engenheira Florestal, Mestre em Ciências Florestais, Universidade Federal do Espírito Santo, UFES, Brasil. E-mail:

<ludpiassi@hotmail.com>.
} 


\section{INTRODUÇÃO}

O manejo florestal moderno requer o conhecimento da floresta e sua interação com o meio, assim como do crescimento e dos produtos que podem ser obtidos para determinados fins (serraria, celulose, chapas, energia etc.). A avaliação florestal e a dinâmica dos povoamentos só podem ser determinadas através de medições de amostras representativas dessas florestas. As principais variáveis utilizadas nesses estudos são o DAP (diâmetro à altura do peito) e a altura total.

A altura constitui-se em importante característica da árvore e pode ser medida ou estimada. Sua medição ou estimação é muito importante para o cálculo do volume, de incrementos em altura e, em determinadas situações, pode servir como indicadora da qualidade produtiva de um local.

Em florestas nativas, a altura total das árvores pode ter importante significado ecológico e para fins de manejo, à medida que ajuda a compreender a estrutura vertical da comunidade. Assim, podem-se estimar, por meio dessa variável, as espécies que apresentam maior importância ecológica, considerando esse tipo de estrutura, tal como discutido por Souza e Souza (2004). A posição sociológica das espécies pode ser calculada tomando-se em conta as alturas das árvores amostradas na comunidade, de acordo, por exemplo, com metodologias apresentadas por Finol (1971), Longhi (1980) e Souza e Leite (1993). Esses autores destacaram a importância em se medir a altura das árvores em estudos fitossociológicos. Segundo Hunter Jr. (1990 citado por SOUZA et al., 2003), nos estratos verticais de uma floresta inequiânea coexistem diferentes grupos de plantas e animais que ocupam diferentes nichos ecológicos. Portanto, a estratificação vertical da floresta influencia a riqueza, a diversidade, o crescimento e a produção de biomassa, sendo importante indicador de sustentabilidade ambiental de uma floresta.

De acordo com Machado e Figueiredo Filho (2003), a altura total de uma árvore pode ser definida como a distância do nível do solo até o topo da árvore, ao longo de seu eixo principal. Diferentemente do diâmetro, a medição da altura não é tarefa tão fácil. Em florestas plantadas homogêneas com espaçamentos regulares, normalmente se medem as alturas de algumas árvores com aparelhos óticos baseados em princípios trigonométricos e a partir da composição de uma amostra que contenha os diâmetros e alturas das árvores, ajustam-se relações hipsométricas que permitirão estimar as alturas das demais árvores. Esse procedimento é muito empregado em florestas homogêneas, como já mencionado, porque diminui o esforço na obtenção das alturas e, em geral, apresenta boa precisão. Entretanto, em povoamentos nativos, as relações hipsométricas não apresentam boa precisão, e outros métodos de se medir a altura são empregados. Para as árvores de até 15 m, podem ser utilizadas as réguas telescópicas. No mercado, encontram-se réguas para esse fim com até $15 \mathrm{~m}$. Essas réguas oferecem uma medida direta da altura e para árvores desse porte podem funcionar muito bem. Entretanto, o rendimento da medição pode diminuir sensivelmente em função da densidade de indivíduos de pequeno porte e do tamanho da régua. Réguas com 15 m de altura já apresentam certa dificuldade de serem manuseadas.

Para árvores maiores que 15 m é comum, em alguns trabalhos, a estimativa visual da altura, com ou sem apoio de uma régua telescópica encostada na árvore. A alternativa seria o uso de hipsômetros. Os hipsômetros baseados em distâncias fixas, como é o caso, por exemplo, dos hipsômetros de Haga e Blume-Leiss, não funcionam bem na estimação da altura em povoamentos nativos, pois a necessidade do mensurador de se afastar a uma distância fixa da árvore muitas vezes faz que ele perca a visão dessa distância. O Hipsômetro Vertex, que emprega impulsos ultrassônicos, permite medir a altura da árvore a qualquer distância, sendo, portanto, opção interessante para se medir a altura em florestas nativas. Pesam contra ele o seu custo mais elevado e as dificuldades para sua manutenção.

Considerando o exposto, foi objetivo deste trabalho comparar alternativas para se estimar a altura total em povoamento florestal nativo, tendo em vista as dificuldades na obtenção dessa variável. Mais especificamente, teve-se como objetivo comparar estimativas visuais e estimativas obtidas com o Hipsômetro Vertex com medidas diretas obtidas das árvores pela sua escalada.

\section{METODOLOGIA}

O Projeto de Pesquisa foi desenvolvido na Reserva Particular do Patrimônio Natural de Cafundó, localizado no distrito de Coutinho, em Cachoeiro de Itapemirim, ES, onde já foi realizado um inventário florestal com 25 parcelas distribuídas de forma sistemática. 
Após selecionar algumas parcelas já mensuradas no inventário, foram escolhidas árvores dentro das seguintes classes de altura total: de 15-25 m (30 árvores) classe 1; 25-35 m (30 árvores) classe 2 e maiores que 35 m (30 árvores) classe 3.

As alturas totais dessas árvores foram estimadas por três métodos diferentes: Hipsômetro Vertex; estimação visual sem treinamento; e estimação visual com treinamento. Além disso, foi realizada a medida direta da altura das árvores por meio de escalada. Para a medição direta com escalada, a altura total foi obtida utilizando-se uma trena. Assim, para maior clareza do leitor, definiram-se os seguintes métodos:

Método 1: Estimação visual sem treinamento;

Método 2: Estimação visual com treinamento; e

Método 3: Estimação com Hipsômetro Vertex.

Desse modo, foram identificados um total de nove tratamentos, tal como apresentado na Tabela 1.

As atividades realizadas em campo para a estimação e medição direta das alturas foram realizadas da seguinte forma: primeiramente foi realizada a estimação da altura da primeira árvore por dois operadores, por meio de avaliação visual. Em seguida foi realizada a estimação da altura com o Hipsômetro Vertex e a escalada na árvore, obtendo-se a altura total real dela. Na sequência, para a medição das próximas árvores, um dos operadores passou a receber treinamento, ou seja, ele era informado sobre a medida real da árvore anterior obtida pela escalada, de modo que pudesse balizar a sua estimativa para as árvores seguintes. Dos dois operadores que fizeram a estimação visual da altura da primeira árvore, a partir da segunda árvore um deles passou a receber treinamento, como já descrito, e o outro continuou

Tabela 1 - Tratamentos avaliados na medição de altura. Table 1 - Treatments evaluated at the height measurement.

\begin{tabular}{ccc}
\hline Tratamento & Método & Classe \\
\hline 1 & 1 & 1 \\
2 & 1 & 2 \\
3 & 1 & 3 \\
4 & 2 & 1 \\
5 & 2 & 2 \\
6 & 2 & 3 \\
7 & 3 & 1 \\
8 & 3 & 2 \\
9 & 3 & 3 \\
\hline
\end{tabular}

a estimar a altura sem qualquer balizamento. Um terceiro operador realizou as medidas com Vertex, e um escalador realizou a medida direta da altura. Cabe ressaltar que a escalada da árvore foi realizada por profissional experiente, usando todos os equipamentos de segurança e tomando o cuidado para que a trena ficasse posicionada da melhor forma possível.

Uma vez obtidas as alturas pelos quatro métodos propostos, foram aplicados testes de médias, no caso o teste $t$ para amostras independentes, em nível de $5 \%$ de probabilidade, para comparar as alturas médias obtidas pelos quatro métodos e os erros médios cometidos para cada método de estimação. Foram realizadas também análises gráficas de resíduos, com o intuito de verificar eventuais tendências na obtenção das alturas. Os valores residuais utilizados na construção dos gráficos foram calculados pela seguinte expressão:

$$
\operatorname{Erro}(\%)=\frac{\mathrm{Y}-\hat{\mathrm{Y}}}{\mathrm{Y}} 100
$$

em que:

$\hat{Y}=$ alturas estimadas; e

$Y=$ alturas observadas obtidas pela escalada.

Foram realizados também testes complementares à análise gráfica dos resíduos, por meio das seguintes estatísticas: Desvio Médio (DM); Média das Diferenças Absolutas (MD); e Desvio-Padrão das Diferenças (DPD), tal como utilizado por Mendonça (2006) e Souza (2007).

A partir da análise das estatísticas $D M, M D$, $D P D$, procedeu-se à ordenação das funções segundo o maior ou menor grau de precisão, sendo atribuídos pesos de 1 a 3 , de acordo com os resultados das estatísticas. Foi considerado o método (tratamento) mais preciso aquele que resultou em menor somatório nas notas.

Os critérios e respectivos estimadores para avaliação dos métodos de estimação de altura (Tratamentos) são calculados pelas seguintes expressões:

Desvio Médio (DM):

$$
D M=\frac{\sum_{i=1}^{n} Y i-\sum_{i=1}^{n} \hat{Y} i}{n}
$$

Revista Árvore, Viçosa-MG, v.36, n.2, p.341-348, 2012 
Média das diferenças absolutas (MD):

$$
M D=\frac{\sum_{i=1}^{n} / Y_{i}-\hat{Y}_{i} /}{n}
$$

Desvio-padrão das diferenças $(D P D)$ :

$$
D P D=\sqrt{\left(\sum_{i=1}^{n} d i^{2}-\left(\sum_{i=1}^{n} d i\right)^{2} / n\right) /(n-1)}
$$

Sendo $Y i=$ altura observada e $\hat{Y} i$ = altura estimada; $n=$ número de observações; e $d i=(Y i-\hat{Y} i)$.

\section{RESULTADOS E DISCUSSÕES}

Pela revisão dos dados do inventário realizado na área do estudo, fez-se uma análise prévia das classes de altura ocorrentes no povoamento florestal estudado, constatando-se que mais de $60 \%$ das árvores se encontram na classe de altura de 5-10 m e aproximadamente $90 \%$ possuem altura menor que $15 \mathrm{~m}$. Esse resultado é favorável à medida que árvores menores que $15 \mathrm{~m}$ possam ser medidas com aparelhos mais precisos e de fácil manuseio. A seguir, são apresentados os resultados relativos às comparações feitas entre os métodos de medição propostos.

\subsection{Comparação estatística dos métodos de medição de altura}

\subsubsection{Teste de média das alturas estimadas por cada método e do erro cometido}

A Tabela 2 apresenta os testes de médias dos tratamentos avaliados, considerando-se a estatística $t$ para amostras independentes, em nível de 5\% de probabilidade. Pela observação dessa tabela, notou-se que os métodos não diferiram estatisticamente entre si, ou seja, os tratamentos T1, T4 e T7, que representam os métodos 1, 2 e 3 na classe 1, foram estatisticamente iguais em nível de $5 \%$ de probabilidade, pelo teste $t$, ou seja, para a classe 1 , tanto fez medir com estimativa visual com ou sem treinamento quanto utilizar o Hipsômetro Vertex, a altura média encontrada foi a mesma. O mesmo ocorreu na classe 2, com os tratamentos T2, T5 e T8; e na classe 3, com os tratamentos T3, T6 e T9. As diferenças estatísticas observadas na Tabela 2 revelam o efeito da classe, ou seja, era esperado que médias estimadas em classes de altura diferentes fossem estatisticamente diferentes, tal como mostra o teste estatístico.

A Tabela 2 permite também avaliar o erro cometido em cada tratamento, considerando-se a altura estimada por cada método em relação à altura verdadeira da árvore (altura obtida por escalada). Nota-se, nesse quadro, que os tratamentos 4 e 6 foram os que proporcionaram erro médio estatisticamente diferente de alguns tratamentos, em nível de $5 \%$ de probabilidade pelo teste $t$. Entretanto, pode-se dizer que, de forma geral, os tratamentos apresentaram erros médios relativos semelhantes.

A Tabela 2 apresenta a comparação dos métodos avaliados entre si. A Tabela 3 permite comparar a altura média estimada em cada tratamento com a altura média real nas respectivas classes, obtida pela escalada da árvore. Nota-se, nessa tabela, que a única diferença encontrada foi no tratamento 6 , ou seja, foi a única situação em que a altura média estimada não coincidiu com a altura real média das árvores. Esse resultado tem alguma coincidência com aquele encontrado na Tabela 2, ou seja, o tratamento 6 apresentou diferença com alguns dos outros tratamentos e, no caso da Tabela 3, com a média das alturas verdadeiras. Os tratamentos 4 e 6 estão ligados ao método de avaliação visual da altura com treinamento na classe 2 , indicando que o treinamento pode não ter funcionado bem nessa classe.

\subsubsection{Análise gráfica dos resíduos}

De acordo com a Figura 1, nota-se que os métodos 1, 2 e 3 apresentaram distribuição residual não tendenciosa na classe 1 de altura. Na classe 2 de altura, nota-se uma leve tendência de subestimativa da altura pelos métodos 1 e 2. Já o método 3 apresentou distribuição residual com pequena tendência de subestimação nas árvores com alturas menores. Na classe 3, observou-se tendência mais clara de subestimativa das alturas estimadas pelos três métodos avaliados. Assim, pode-se conjecturar com base nos resultados encontrados que, de maneira geral, avaliando as análises gráficas das classes de altura consideradas (classes 1, 2 e 3), a tendência aumenta à medida que também aumentam as classes de altura. Isso sugere que os métodos de estimação de altura avaliados apresentaram estimativas mais confiáveis para a menor classe de altura (classe 1) e menos confiáveis para a maior classe de altura (classe 3 ). 
Tabela 2 - Comparação entre os tratamentos avaliados nas diferentes classes, tal como definido na Tabela 1, considerando-se as variáveis altura total média e erro relativo.

Table 2 - Comparison among the treatments evaluated in the different classes, as defined in the Table 1, considering the variables average total height and relative error.

\begin{tabular}{|c|c|c|c|c|c|c|c|c|c|}
\hline \multicolumn{10}{|c|}{ Estimativa da altura da árvore } \\
\hline & $\mathrm{T} 1$ & $\mathrm{~T} 2$ & $\mathrm{~T} 3$ & $\mathrm{~T} 4$ & $\mathrm{~T} 5$ & $\mathrm{~T} 6$ & $\mathrm{~T} 7$ & $\mathrm{~T} 8$ & T9 \\
\hline $\mathrm{T} 1$ & - & & & & & & & & \\
\hline $\mathrm{T} 2$ & $* *$ & - & & & & & & & \\
\hline $\mathrm{T} 3$ & $* *$ & $* *$ & - & & & & & & \\
\hline $\mathrm{T} 4$ & ns & $* *$ & $* *$ & - & & & & & \\
\hline T5 & $* *$ & ns & $* *$ & $* *$ & - & & & & \\
\hline T6 & $* *$ & $* *$ & ns & $* *$ & $* *$ & - & & & \\
\hline $\mathrm{T} 7$ & ns & $* *$ & $* *$ & ns & $* *$ & $* *$ & - & & \\
\hline $\mathrm{T} 8$ & $* *$ & ns & $* *$ & $* *$ & $\mathrm{~ns}$ & $* *$ & $* *$ & - & \\
\hline T9 & $* *$ & $* *$ & ns & $* *$ & $* *$ & ns & $* *$ & $* *$ & - \\
\hline \multicolumn{10}{|c|}{ Erro relativo } \\
\hline & $\mathrm{T} 1$ & $\mathrm{~T} 2$ & $\mathrm{~T} 3$ & $\mathrm{~T} 4$ & T5 & $\mathrm{T} 6$ & $\mathrm{~T} 7$ & $\mathrm{~T} 8$ & T9 \\
\hline $\mathrm{T} 1$ & - & & & & & & & & \\
\hline $\mathrm{T} 2$ & ns & - & & & & & & & \\
\hline T3 & $\mathrm{ns}$ & $\mathrm{ns}$ & - & & & & & & \\
\hline $\mathrm{T} 4$ & ns & $\mathrm{ns}$ & $* *$ & - & & & & & \\
\hline T5 & ns & ns & ns & $* *$ & - & & & & \\
\hline T6 & $* *$ & ns & ns & $* *$ & ns & - & & & \\
\hline T7 & ns & ns & ns & ns & ns & ns & - & & \\
\hline T8 & ns & ns & ns & $* *$ & ns & $\mathrm{ns}$ & ns & - & \\
\hline T9 & $\mathrm{ns}$ & ns & ns & $* *$ & ns & $\mathrm{ns}$ & ns & ns & - \\
\hline
\end{tabular}

** Significativo a 5\% de probabilidade, pelo teste $t$.

ns Não significativo a $5 \%$ de probabilidade, pelo teste $t$.

Tabela 3 - Comparação entre as médias de cada tratamento com a respectiva média da altura verdadeira em cada uma das classes.

Table 3 - Comparison among the averages of each treatment with the respective real height average in each one of the classes.

\begin{tabular}{cc}
\hline Tratamento & Nível de significância \\
\hline 1 & ns \\
2 & ns \\
3 & ns \\
4 & ns \\
5 & ns \\
6 & $* *$ \\
7 & ns \\
8 & ns \\
9 & ns \\
\hline
\end{tabular}

** Significativo a 5\% de probabilidade, pelo teste $t$. ns Não significativo a $5 \%$ de probabilidade, pelo teste $t$.

\subsubsection{Testes de precisão dos métodos}

O Quadro 4 apresenta as estatísticas Desvio Médio $(D M)$, Média das Diferenças Absolutas (MD) e Desvio-
Padrão das Diferenças ( $D P D)$ das estimativas de altura pelos diferentes métodos nas classes 1,2 e 3 . Os valores positivos e negativos da estatística $D M$ indicam subestimativa e superestimativa, respectivamente. Os menores valores das três estatísticas testadas indicam que o método de estimação de altura apresenta maior precisão.

A Tabela 4 mostra também as notas atribuídas para as estimativas de altura referentes às classes 1 , 2 e 3, tomando-se com base as estatísticas $D M$, $M D$ e $D P D$. No caso da estatística $D M$, a menor nota foi atribuída para a classe que apresentou resultado mais próximo de zero, independente de ser positivo ou negativo.

Tomando como exemplo o método 2 para a classe 1 , ou seja, o tratamento 4, apresentou valor de $D P D$ de 2,7583 . Considerando que este foi o menor valor e comparado com o DPD dos outros métodos de estimação de altura, a nota atribuída a esse método nessa classe de altura foi 1 . Esse valor significa que, considerando o DPD, o método 2 obteve a melhor estimativa em relação

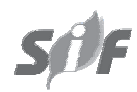

Revista Árvore, Viçosa-MG, v.36, n.2, p.341-348, 2012 
Classe 1

Sem treinamento

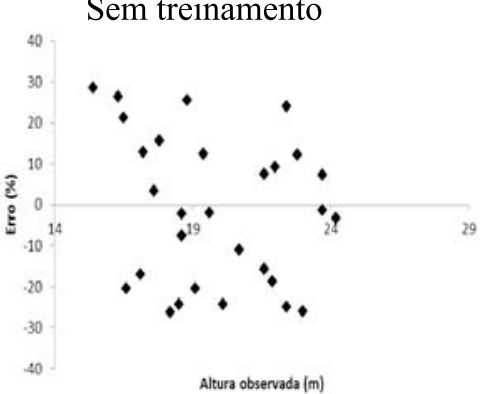

Com treinamento

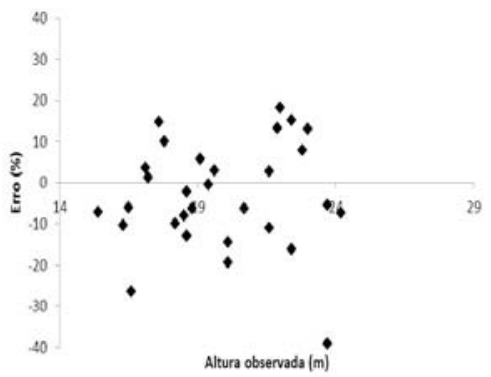

Vertex

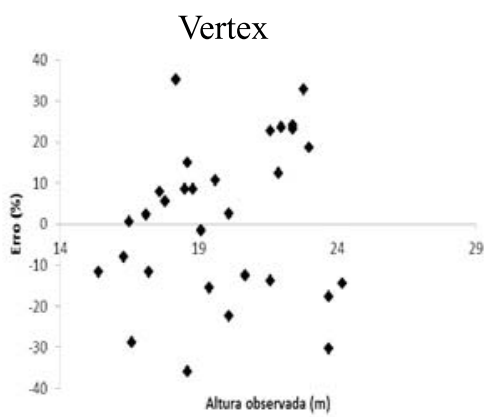

Classe 2
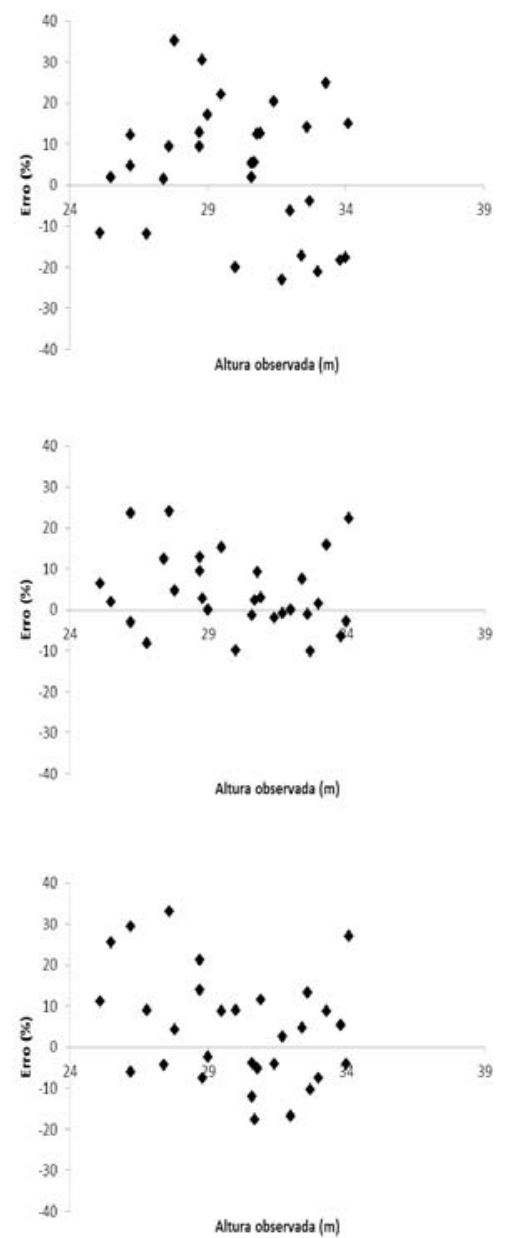

Classe 3
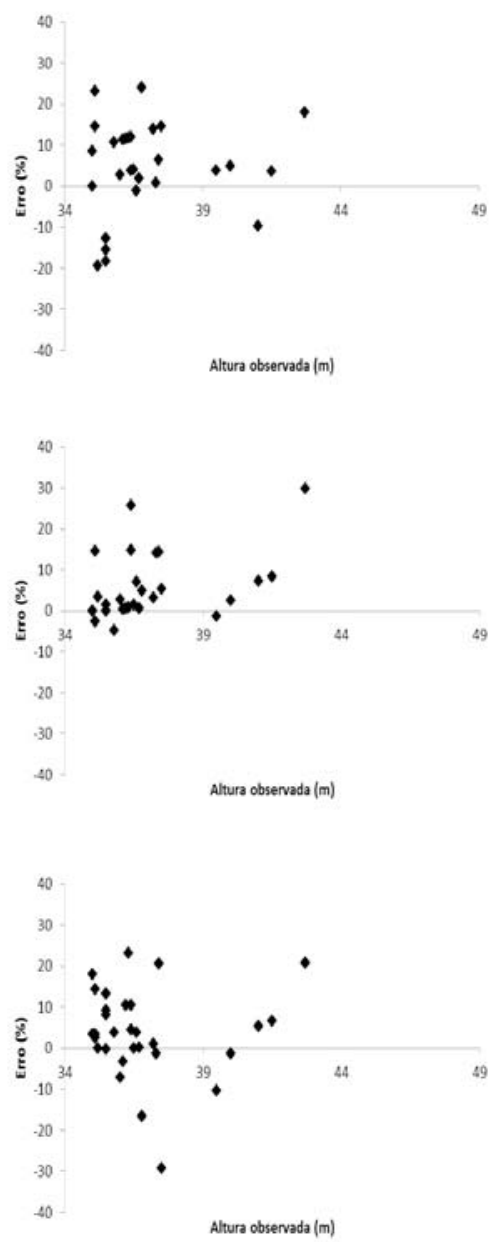

Figura 1 - Distribuição dos resíduos de altura, em porcentagem, para os métodos 1, 2 e 3, nas três classes de altura. Figure 1 - Distribution of the height residuals, in percentage, for the methods 1, 2 and 3 for the three height classes.

às outras estimativas avaliadas, seguida, pela ordem, pelos métodos 1 (nota 2 ) e 3 (nota 3 ).

Seguindo o raciocínio apresentado e analisando os resultados mostrados na Tabela 4, verificou-se que na classe 1 de altura o método 2 apresentou o melhor resultado, seguido pelos métodos 1 e 3 , respectivamente. Provavelmente, o pior desempenho do método 3 para estimação de altura na classe 1 (15-25 m) se justifica pela maior dificuldade em visualizar a copa da árvore a ser avaliada, visto que se trata de uma floresta nativa, e esse fato pode ser um dos responsáveis pelos erros apresentados.

Revista Árvore, Viçosa-MG, v.36, n.2, p.341-348, 2012
Para a avaliação da altura pelos métodos 1 e 2, os mensuradores conseguem visualizar melhor a copa, pois, no caso de dificuldade em vê-la a certa distância, o mensurador pode se aproximar, conseguindo, ainda assim, fazer a estimação. Já no caso do método 3, ao se posicionar a pequenas distâncias da árvore, o ângulo de inclinação do Vertex em relação à copa é aumentado, fazendo que pequenas oscilações no momento da leitura correspondam a erros mais significativos.

Na classe 2 de altura, o método 2 também apresentou melhor resultado, havendo empate em termos de precisão entre os métodos 1 e 3 , tendo em vista as estatísticas 
Tabela 4 - Notas atribuídas a cada tratamento com base nas estatísticas Desvio Médio (DM), Média das Diferenças Absolutas $(M D)$ e Desvio-Padrão das Diferenças ( $D P D)$.

Table 4 - Notes attributed to each treatment with base in the statistics Average Deviation (DM), Average of the Absolute Differences (MD) and Standard Deviation of the Differences (DPD).

\begin{tabular}{|c|c|c|c|c|c|c|c|c|c|}
\hline Métodos & Classe & Tratamento & $D M$ & $M D$ & $D P D$ & $D M$ & $M D$ & $D P D$ & Total \\
\hline & 1 & 1 & $-0,5167$ & 3,0833 & 3,5277 & 2 & 2 & 2 & 6 \\
\hline \multirow[t]{4}{*}{ Sem treinamento } & 2 & 2 & 1,0967 & 4,2633 & 4,9319 & 1 & 3 & 3 & 7 \\
\hline & 3 & 3 & 1,6133 & 3,8267 & 4,2942 & 2 & 3 & 3 & 8 \\
\hline & Total & - & - & - & - & 5 & 8 & 8 & 21 \\
\hline & 1 & 4 & $-0,6503$ & 2,1567 & 2,7583 & 3 & 1 & 1 & 5 \\
\hline \multirow[t]{4}{*}{ Com treinamento } & 2 & 5 & 1,2312 & 2,2033 & 2,8019 & 2 & 1 & 1 & 4 \\
\hline & 3 & 6 & 1,9856 & 2,2467 & 3,1652 & 3 & 1 & 1 & 5 \\
\hline & Total & - & - & - & - & 8 & 3 & 3 & 14 \\
\hline & 1 & 7 & 0,2733 & 3,2667 & 3,9499 & 1 & 3 & 3 & 7 \\
\hline \multirow[t]{3}{*}{ Vertex } & 2 & 8 & 1,2533 & 3,3600 & 3,9695 & 3 & 2 & 2 & 7 \\
\hline & 3 & 9 & 1,3933 & 3,1333 & 4,1188 & 1 & 2 & 2 & 5 \\
\hline & Total & - & - & - & - & 5 & 7 & 7 & 19 \\
\hline
\end{tabular}

consideradas. A aparente melhora de desempenho do método 3 na verdade pode ser mais bem explicada pela piora do método 1, ou seja, seria ilusório pensar que para árvores maiores o Vertex passou a funcionar melhor. O que parece ter ocorrido é que, em árvores menores (classe 1), utilizar o Vertex consiste em tarefa difícil pela característica do aparelho e da floresta, o que obriga o mensurador a ficar próximo da árvore, e as oscilações no momento da medida podem provocar erros maiores. Como a estimação visual não depende de aparelhos, nas árvores menores as chances de o mensurador ser mais preciso são maiores, o que pode explicar o pior desempenho do Vertex na classe 1 em relação à estimação visual. Com o aumento do tamanho das árvores, as dificuldades no uso do Vertex aumentam, mas a limitação no uso do aparelho continua basicamente a mesma. Já a avaliação visual parece ter sofrido impacto maior em termos de perda de precisão. Isso pode ser explicado pelo fato de que, em árvores maiores, as referências para o avaliador visual ficam bem mais difíceis, de modo que ele possa comparar a árvore com uma altura que ele tem como referência mental e definir a altura estimada da árvore.

Quando se observam na Tabela 4 os resultados na classe 3, a tese anteriormente apresentada se reforça, ou seja, há perda considerável de precisão no método 1 . Entretanto, o Vertex também perde em precisão com o aumento da altura, mas isso não acontece tão rapidamente como no método 1 , fazendo que nas árvores maiores o Vertex tenha tido desempenho superior ao da avaliação sem nenhum treinamento.

É interessante observar que, quando se compara o Vertex com a estimação com treinamento, a perda de precisão no método com treinamento não acontece de forma tão abrupta com o aumento da altura. Observando a Tabela 4, verifica-se que esse método demonstrou ser mais preciso que o Vertex na classe 2 e teve o mesmo desempenho na classe 3. Assim, o treinamento parece ter sido eficaz a ponto de indicar que o Vertex e a avaliação com treinamento produzem resultados semelhantes em termos de precisão na estimação das alturas.

É importante ressaltar que o mensurador que realizou as medições de altura com treinamento é inexperiente nessa tarefa, e o treinamento que ele recebeu se restringiu a esse experimento, ou seja, não pode ser considerado intenso. Mesmo assim, o treinamento parece ter sido eficaz, como demonstrado pelos resultados. Admitindo-se que um técnico receba treinamento mais intenso e qualificado e que, ao longo do tempo, ganhe experiência nessa tarefa, é razoável pensar que o método de estimação visual da altura com treinamento seja opção promissora para ser utilizada em florestas naturais com problema semelhante ao estudado neste trabalho.

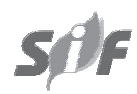

Revista Árvore, Viçosa-MG, v.36, n.2, p.341-348, 2012 
Segundo Bechtold et al. (1998) e Brack (1999), pode-se esperar aumento no nível de precisão da estimativa ocular, desde que as pessoas responsáveis pelas estimativas sejam treinadas de forma mais intensiva e realizem “calibração” em cada parcela a ser inventariada, através da medição prévia de algumas árvores com aparelhos de medição de altura. Torna-se evidente que, apesar da utilização de instrumentos óticos de alta precisão, como é o caso do Vertex, é necessário dispor de pessoas que possam estimar visualmente a altura das árvores com razoável precisão (BECHOTOLD et al., 1998; BRACK, 1999; FORSTREUTER; WAKOLO, 2000).

\section{CONCLUSÕES}

Os resultados possibilitaram as seguintes conclusões:

O aumento na altura da árvore comprometeu o desempenho da estimação dessa variável em termos de precisão nos três métodos avaliados. Esse comprometimento foi mais evidente no método 1 , ou seja, estimação visual da altura sem nenhum treinamento.

A única diferença estatística, a de 5\% de probabilidade pelo teste $t$, entre as médias das alturas dos três métodos avaliados e as médias verdadeiras, considerando-se as três classes testadas, ocorreu no método de estimação visual com treinamento na classe 2 .

As estatísticas Desvio Médio ( $D M)$, Média das Diferenças Absolutas (MD) e Desvio- Padrão das Diferenças $(D P D)$ indicaram, considerando-se as três classes de altura avaliadas, maior precisão na estimação da altura do método com treinamento, seguido pelo Vertex e estimação visual sem treinamento.

\section{REFERÊNCIAS}

BECHTOLD, W. A.; ZARNOCH, S. J.; BURKMAN, W. G. Comparisons of modeled height predictions to ocular height estimates. 1998. Disponível em: < http:// www.srs.fs.fed.us/pubs/ viewpub.jsp.index =439> . Acesso em: 24 nov. 2007.

BRACK, C. Ocular estimation of height. 1999 Disponível em: <http://online.anu.edu.au/Foresty/ mensuration/toolshd.htm >. Acesso em: 24 nov. 2007.
FINOL, U. H. Nuevos parâmetros a considerase em $\mathrm{El}$ analisis estrutural de las selvas virgenes tropicales. Revista Florestal Venezuelana, v.14, n.21, p.29-42, 1971.

FROSTREUTER, W.; WAKOLO, J. Remote sensing for tree measurements in Fiji. , Fiji: South Pacific GIS \& Remote Sensing News, 2000.p.1-2.

HUNTER JR., M. L. Wildlife forests, and forestry: principles of managing forests for biological diversity. New Jersey: Prentice-Hall, 1990. 370p.

LONGHI, S. J. A estrutura de uma floresta natural de Araucaria angustifólia (Bert.) O. Ktze, no sul do Brasil. 1980. 198f. Dissertação (Mestrado em Engenharia Florestal) Universidade Federal do Paraná, Curitiba, 1980.

MACHADO, S. A.; FIGUEIREDO FILHO, A. Dendrometria. Curitiba: Universidade Federal do Paraná, 2003. 309p.

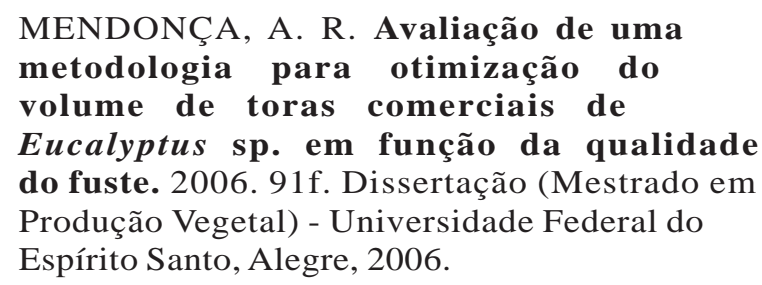

SOUZA, A. L.; LEITE, H. G. Regulação da produção em florestas inequiâneas. Viçosa, MG: Universidade Federal de Viçosa, 1993. 147p.

SOUZAA. L. et al. Emprego de análise multivariada para estratificação vertical de florestas ineqüiâneas. Revista Árvore, v.27, n 1, p.59-63, 2003.

SOUZA A. L.; SOUZA, D. R. Estratificação vertical em floresta ombrófila densa de terra firme não explorada, Amazônia Oriental. Revista Árvore, v.28, n.5, p.691-698, 2004.

SOUZA, C. A. M. Avaliação de modelos de taper não segmentados e segmentados na estimação da altura e volume comercial de fustes de Eucalyptus sp. 2007. 94f. Dissertação (Mestrado em Produção Vegetal) Universidade Federal do Espírito Santo, Alegre, 2007. 